Research Article

\title{
Sound Radiation Analysis of a Front Side Window Glass of DrivAer Model under Wind Excitation
}

\author{
Yinzhi He $\mathbb{D}^{1,2}$ Zihao Shi ${ }^{1},^{1,2}$ Yu Wu ${ }^{1 D},^{1,2}$ and Zhigang Yang ${ }^{1,2}$ \\ ${ }^{1}$ School of Automotive Studies, Tongji University, Shanghai 201804, China \\ ${ }^{2}$ Shanghai Key Lab of Vehicle Aerodynamics and Vehicle Thermal Management Systems, Tongji University, \\ Shanghai 201804, China \\ Correspondence should be addressed to Yinzhi He; heyinzhi@tongji.edu.cn
}

Received 11 February 2018; Revised 13 July 2018; Accepted 14 August 2018; Published 2 October 2018

Academic Editor: Hamid Hosano

Copyright (c) 2018 Yinzhi He et al. This is an open access article distributed under the Creative Commons Attribution License, which permits unrestricted use, distribution, and reproduction in any medium, provided the original work is properly cited.

To study the car radiated noise caused by turbulent pressure fluctuation on the side glass under wind excitation, a clay model of DrivAer was constructed except that the front left side window was built with real glass. Firstly, the constraint boundary condition of the side glass was set equivalent to a series of springs for the modelling with Finite Element Method (FEM). Then, a platform based on Matlab-Abaqus cosimulation was originally developed, and the genetic algorithm was applied to find out the best fitted spring stiffness, which was used to build the equivalent model. Then working as excitation, the Corcos model was applied to calculate the power spectrum density of the turbulence pressure fluctuations acting on the side glass. Subsequently, the finite element modal superposition method was used to solve the vibroacoustic coupling equation to get the noise level of the driver's ear position in the vehicle interior. Finally, the surface vibration velocity distribution of side glass under wind excitation was measured with Laser Vibrometer and then with Boundary Element Method (BEM), the radiated noise into interior was calculated as well (semisimulation). Through comparison of these two results, it shows good agreement up to $1000 \mathrm{~Hz}$. It demonstrates that the above method is applicable to calculate the sound radiation caused by the side glass' vibration at the low and middle frequency range. Therefore, an approach of calculating sound radiation of a vibrating glass caused by the air convective pressure fluctuation was explored.

\section{Introduction}

Besides leakage noise, the turbulence induced noise and pressure fluctuation transmitted into vehicle interior through the front side window glass is a major contributor to noise level at the driver's ear position by the external wind excitation at high speed [1]. The pressure fluctuation on the side glass surface mainly comprises two parts: convective pressure fluctuation and acoustic pressure fluctuation. Normally, the energy of convective pressure fluctuation is mainly concentrated at the low frequency range, which can excite the side glass to vibrate and hence radiate noise into vehicle interior. However, the energy of turbulence-induced acoustic pressure fluctuation is mainly concentrated at the high frequency range and can be transmitted into vehicle interior with certain transmission loss through the glass. These two kinds of noises have different generation and transmission mechanisms. Study separately on their generation and transmission processes is helpful for the vehicle interior aerodynamic noise controlling and therefore for the improving of ride comfort.

There are several ways to calculate vehicle interior aerodynamic noise under wind excitation, such as FEM, BEM, and Statistical Energy Analysis (SEA). At the low frequency range, the structure vibration modes are deterministic, so FEM is an effective tool for the simulation with accuracy. As analysis frequency goes up, BEM can be more suitable for the simulation. While at the high frequency range, mode density becomes higher, and the SEA method should be applied. This study mainly focuses on the convective pressure fluctuation induced vehicle interior noise; hence, FEM and BEM are applied for the calculation work.

The DrivAer model was employed in this study, which was developed by Technical University of Munich and car 
manufacturers Audi and BMW in Germany in recent years. The exterior styling of DrivAer model was derived by interpolation from production series of Audi A4 and BMW3 with the typical characteristics of a real vehicle, but without front-end air intake system. The DrivAer model offers three different kinds of back styling: estate back, notch back, and fast back. In this study, a full-scale clay model was built according to the DrivAer model with notch back, as shown in Figure 1. Since the focus of the study is the relationship of the vehicle exterior flow and interior noise through transmission of the side glass without consideration of vehicle sealing system, a real glass with $4 \mathrm{~mm}$ thickness was adopted and fixed with glue in the front left window frame, while the rest parts of the model were covered with thick clay, so that they can be considered as rigid parts without vibration but with great sound transmission loss. In this way, possibility of transmission for the exterior noise and vibration through other parts into vehicle interior can be excluded. Hence, sound transmission study can be concentrated purely on the front side glass. In the vehicle interior, an isolated cabin of the driver's space was constructed with rigid walls, so that a microphone can be assumed to set near the driver's ear position to apperceive the noise.

About vehicle aerodynamic noise concerning side window glass, some researchers have already done some work in recent years. In 2015, Hartmann et al. [2] studied the separation of convective and acoustic pressure fluctuations using wavenumber-frequency spectrum as a filter on the side glass of a simplified SAE model. In the same year, Schell and Cotoni [3] calculated the interior noise using CFD and vibroacoustic methods. An accurate excitation field was calculated with a hybrid computational aeroacoustics (CAA) approach based on an incompressible unsteady flow field with an acoustic wave equation. From 2014 to 2016, Blanchet et al. [4, 5] studied the characteristics of pressure fluctuations on the side glass. At first, Corcos model was used to characterize the convective part, and propagating wavefield was used to characterize the acoustic part. Later, the method was further improved, and a complex turbulent cross-correlation function was proposed to characterize the pressure fluctuations. The function was applied to a SEA side glass and the interior wind noise was obtained. However, up to now, the research work related with side glass mainly focuses on the total interior noise level and its verification with experiment, little work was found to present the constitution of the different parts of the interior noise. This paper focuses solely on the convective pressure fluctuation induced vehicle interior noise and its experimental verification.

Some research work has been done with DrivAer model. In 2012, Heft et al. [6] studied the aerodynamic characteristics of DrivAer model with experimental and numerical methods and focused on the development of this model. In 2017, using 3 different configurations of this model, Soares et al. [7] studied the formation process of wake vortices in the far-field. Up to now, the research of the DrivAer model mostly focuses on the aerodynamics, no study about aeroacoustics of this model was found yet.

In this paper, the constraint boundary condition of the side glass was set equivalent to a series of springs to get an

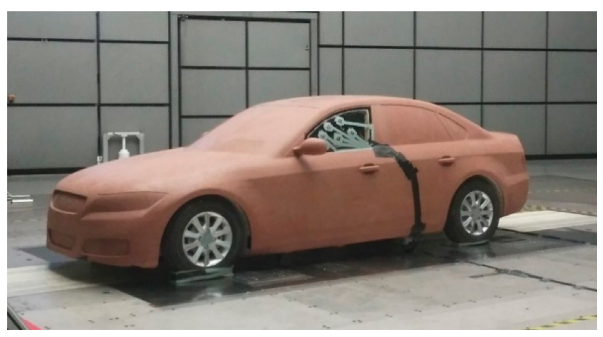

FIgURE 1: DrivAer clay model with notch back.

equivalent model. Then an optimization platform based on Matlab-Abaqus cosimulation was developed originally and the genetic algorithm was applied to find out the optimized spring stiffness for the equivalent model. Subsequently, with the empirical formula of Corcos model, the approximate Power Spectrum Density (PSD) of the pressure fluctuations as excitation on the side glass was formed, and the vibroacoustic coupling equation was solved with finite element modal superposition method to obtain the aerodynamic noise near the driver's ear position. Finally, the surface vibration velocity distribution of the side glass under wind excitation was measured with Laser Vibrometer. And then with BEM, the radiated interior noise was calculated as well (semisimulation). Through comparison of the simulation and semisimulation results, the applicability of using constraint boundary equivalent model, Corcos model and modal superposition method to get the radiated noise from the vibrating glass under wind excitation could be verified. And in this way, the approach of calculating sound radiation of a vibrating glass caused by the convective pressure fluctuation could be explored.

\section{Modelling of Constraint Boundary Condition of the Side Glass}

The side glass was fixed in the clay model with glue. Since the stiffness of the supporting body frame is not rigid enough and the test model was supported with tires on the ground, the constraints of the side glass can't be considered as completely fixed, but with some degrees of freedom (DOFs). Furthermore, wind excitation could force the clay model to move as rigid body displacement and swaying. The displacement is small and can be regarded as linear deformation, so the constraints around the side glass can be regarded as linear elastic constraints. Hence, the study of the side glass boundary constraints can be equivalent to the study of the spring stiffness. To find out the equivalent spring stiffness, the deviation of the first three natural frequencies derived from experimental modal analysis and FEM simulation of the side glass was treated as the optimization target. Through the developed Matlab-Abaqus cosimulation platform and genetic algorithm, the fitted spring stiffness could be obtained.

2.1. Modal Test Analysis. To obtain the dynamic characteristics of the test vehicle's front side glass and to provide the optimization target for fitting the spring stiffness of the 
equivalent model, the side glass under the real boundary constraint was tested with hammer impact method. This test was carried out with multi-point excitation and single point response. 35 excitation points were arranged on the surface of the front side glass averagely. A response point was set at point 29 in the lower right corner of the window. During the test, an accelerometer was pasted at point 29 to collect the acceleration response signal of the side glass under the excitation of an impulse force from a hammer hit. Test equipment and measuring points are shown in Figures 2 and 3.

Data sampling system from HEAD acoustics $\mathrm{GmbH}$ was used to acquire the test data. All the frequency response functions of the side glass collected in the experiment were fitted to obtain the modal parameters of the system. Table 1 shows the first three natural frequencies and damping ratio, and Figure 4 shows the corresponding first three mode shapes.

2.2. Establishment of an Equivalent Model. The width of the seal around the side glass is about $35 \mathrm{~mm}$. When the real constraint was considered to be replaced with series of spring supporting, an equivalent finite element model could be obtained. As shown in Figure 5, the nodes in red are spring supporting. To simulate the most suitable spring stiffness of side glass constraint, the Matlab-Abaqus cosimulation platform was developed to find out the optimal solution of spring stiffness, based on genetic algorithm.

2.2.1. Control Target. To simulate the real side window boundary constraint and obtain the optimal spring stiffness, the standard deviation of the natural frequencies is taken as the control target, since natural frequency is the inherent characteristic of a system. The definition is shown in Equation (1). It can be seen from the equation, the closer the standard deviation is to zero, the better are the optimization results.

$$
S=\sqrt{\frac{\sum_{i=1}^{n}\left(X_{i}-X_{i}^{\prime}\right)^{2}}{n}}
$$

where $S$ is the standard deviation of the natural frequencies, $X_{i}$ is the simulation value of the ith natural frequency, $X_{i}^{\prime}$ is the test value of the $i^{\text {th }}$ natural frequency, and $n$ is the number of samples, here the first 3 nature frequencies are taken into account.

\subsubsection{Establishment of Matlab-Abaqus Cosimulation} Platform. Some commercial FEM software such as Abaqus is very powerful, but most of them does not contain optimization function or only with one certain algorithm. Therefore, a Matlab-Abaqus cosimulation platform was explored in this paper. With the help of this platform, some more optimization algorithms could be compiled in Matlab and combined with the finite element model to form a close loop to find out the optimal solution with high efficiency.

The main idea of using Matlab-Abaqus cosimulation platform for side glass spring stiffness fitting is using Matlab to modify Abaqus finite element model (INP file) and calling Abaqus/cae for calculation. When Abaqus calculation is finished, the calculation results are returned to Matlab. Then

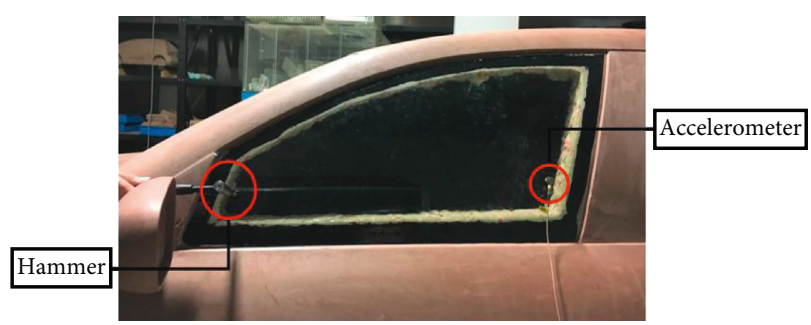

Figure 2: Modal test for the side glass.

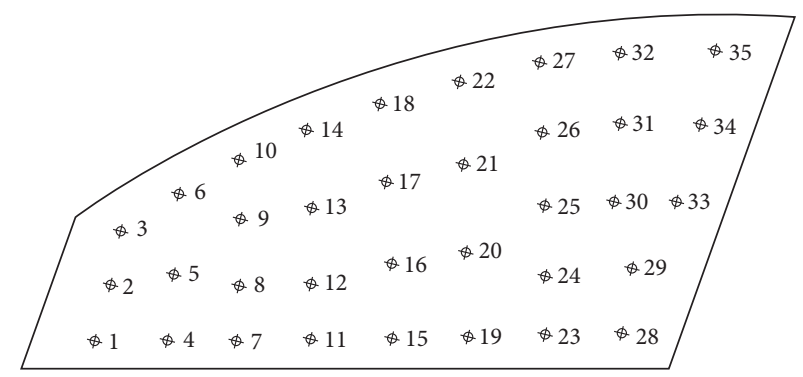

FIGURE 3: Location of measurement points for modal test.

TAвLE 1: First three natural frequencies and damping ratio of the side glass.

\begin{tabular}{lcc}
\hline Mode no. & Natural frequency $(\mathrm{Hz})$ & Damping ratio $(\%)$ \\
\hline 1 & 301 & 7.49 \\
2 & 498 & 4.91 \\
3 & 678 & 4.86 \\
\hline
\end{tabular}

genetic algorithm code in Matlab is applied to do iterative calculations. Finally, the best spring stiffness can be fitted. In the fitting process, Matlab cannot extract directly the results from Abaqus for standard deviation calculation because it cannot recognize the result of ODB file generated by Abaqus calculation. However, the ODB file can be read out by the Python language, so the Python script is called by Matlab to read Abaqus calculation results and get the data. The flowchart is shown in Figure 6.

As original, the spring stiffness ranged from $5000 \mathrm{~N} / \mathrm{mm}$ to $10000 \mathrm{~N} / \mathrm{mm}$ by trial and error; then, the best spring stiffness with the value of $8124.82 \mathrm{~N} / \mathrm{mm}$ was obtained by using above mentioned optimization method. The fitness result of standard deviation of the natural frequencies is shown in Figure 7. With the optimized stiffness value, the simulation work could be done.

2.3. Results Verification. To verify the reliability of the equivalent method for the constraint boundary condition of the side glass, the results between experiment and simulation were compared. Table 2 shows the comparison of natural frequencies between test and simulation result. Although only the first three natural frequencies were used as the control target, the first five natural frequencies below $1000 \mathrm{~Hz}$ derived from test and simulation are all in good agreement, as Table 2 shows. Figure 8 shows the comparison of the first three mode 


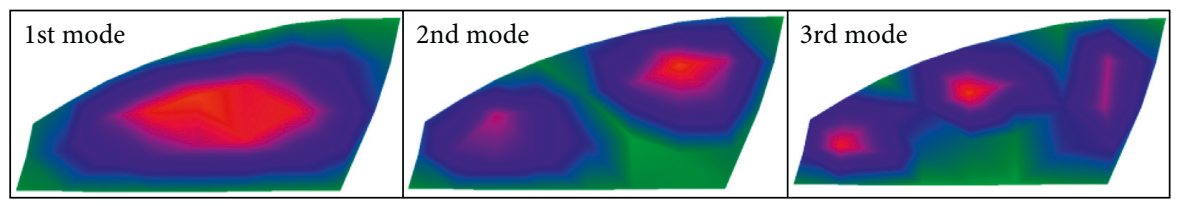

Figure 4: First three mode shapes of the side glass.

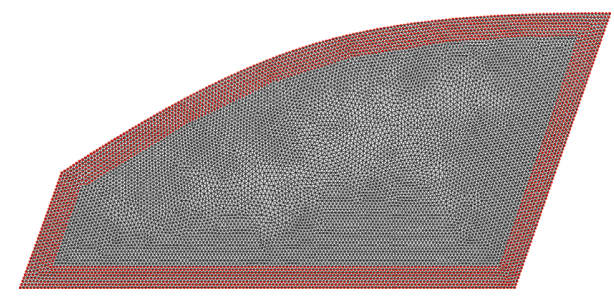

Figure 5: Finite element model of the side glass.

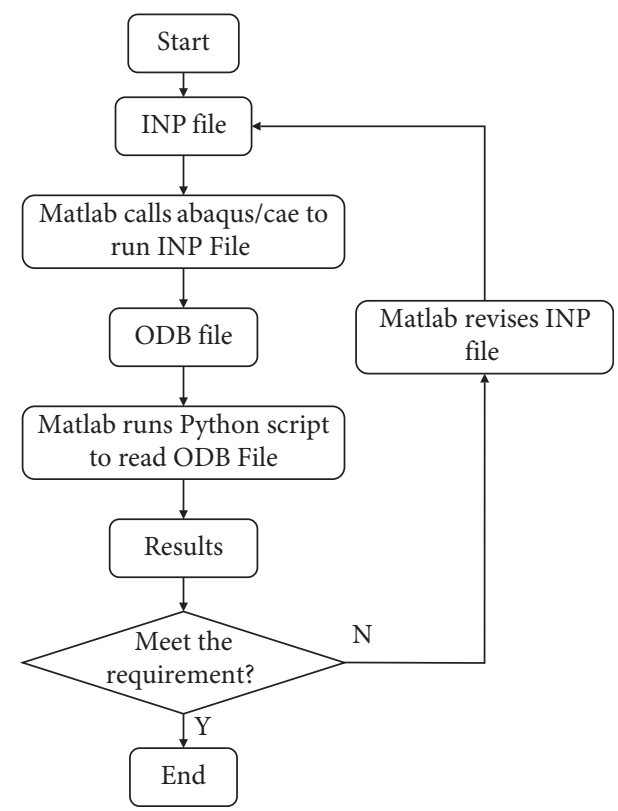

Figure 6: Matlab-Abaqus cosimulation process.

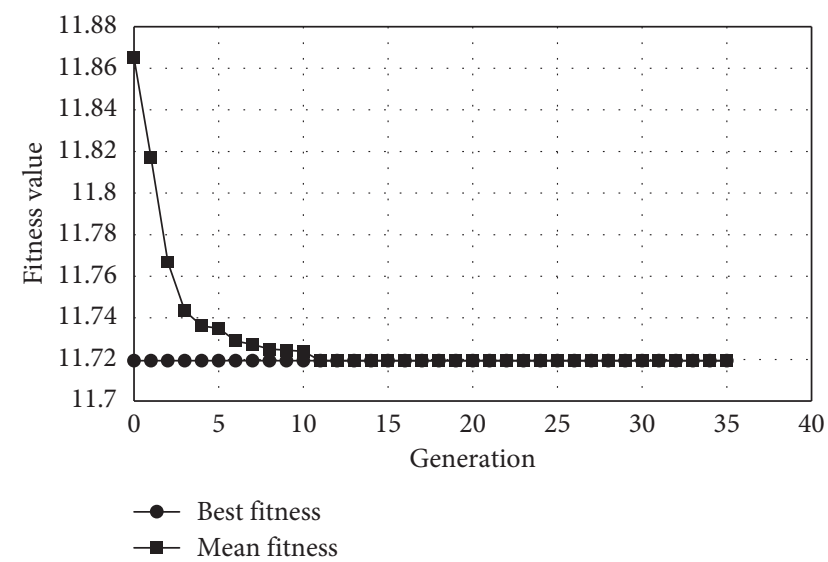

Figure 7: Fitness result of standard deviation of the natural frequencies.
TABLE 2: Comparison of the first 5 natural frequencies between test and simulation.

\begin{tabular}{lccc}
\hline $\begin{array}{l}\text { Mode } \\
\text { no. }\end{array}$ & $\begin{array}{c}\text { Tested natural } \\
\text { frequency }(\mathrm{Hz})\end{array}$ & $\begin{array}{c}\text { Simulated } \\
\text { natural } \\
\text { frequency }(\mathrm{Hz})\end{array}$ & $\begin{array}{c}\text { Relative } \\
\text { error }(\%)\end{array}$ \\
\hline 1 & 301 & 326 & 8.3 \\
2 & 498 & 505 & 1.4 \\
3 & 678 & 668 & 1.5 \\
4 & 853 & 848 & 0.6 \\
5 & 929 & 935 & 0.6 \\
\hline
\end{tabular}

shapes as example. It is obvious that the mode shapes of the same order have good agreement. It can be seen that the first mode shape has a single respiratory cavity, the second mode shape has two respiratory cavities with the opposite phase, and the third mode shape has three respiratory cavities.

Normally, Modal Assurance Criterion (MAC) is used to characterize the correlation between tested and simulated mode shapes. The equation is described as follows:

$$
\text { MAC }=\frac{\left|\Phi_{\mathrm{e}}^{h} \cdot \Phi_{\mathrm{s}}\right|^{2}}{\Phi_{\mathrm{e}}^{h} \cdot \Phi_{\mathrm{e}} \cdot \Phi_{\mathrm{s}}^{h} \cdot \Phi_{\mathrm{s}}},
$$

where $\Phi_{\mathrm{e}}$ is the modal shape vector of experiment, $\Phi_{\mathrm{s}}$ is the modal shape vector of simulation, and " $h$ " represents the conjugate transpose transform. Usually when MAC value is greater than 0.7, the two shape vectors can be considered linearly correlated. And when MAC value is less than 0.2, vectors can be considered orthogonal.

Figure 9 shows the MAC values bar for the tested and simulated modal shapes.

It can be seen from the MAC values of the first and second modal shapes from the tested and simulated results are linearly correlated. However, the linear correlation of the third modal shape is not very good. Through comparison of the third modal shape, it shows that there exists a location deviation in the middle respiratory cavity, and this results in a decrease of the MAC value. However, generally speaking, the equivalent model is basically consistent with the actual boundary conditions and can be used for the later work.

Through above comparison, it was proved that the equivalent spring stiffness fitting method based on the Matlab-Abaqus cosimulation platform and genetic algorithm is reasonable and effective.

\section{Calculation of the Sound Pressure Level near the Driver's Ear Position}

The air pressure fluctuation acting on the side glass excites the glass to vibrate, and through the vibration, noise can be 


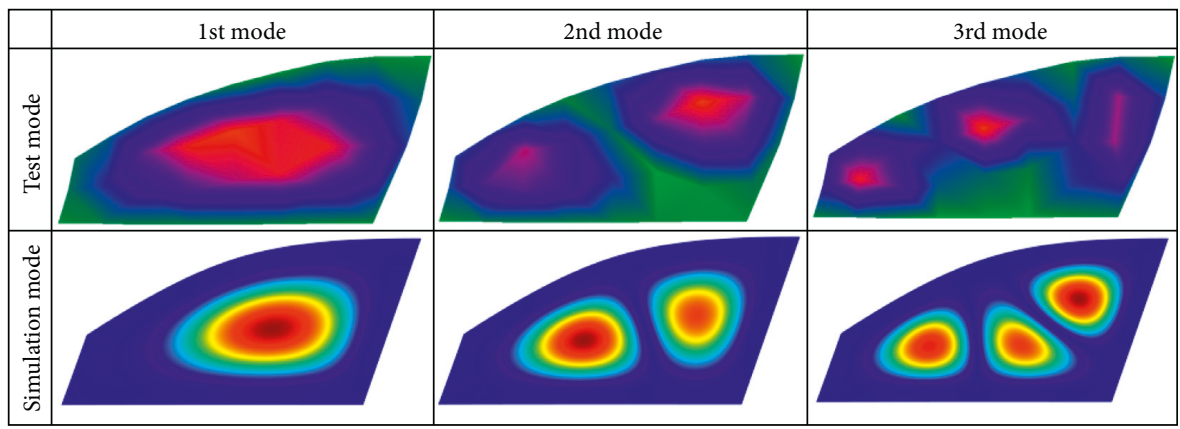

Figure 8: Comparison of the first 3 mode shapes from test and simulation.

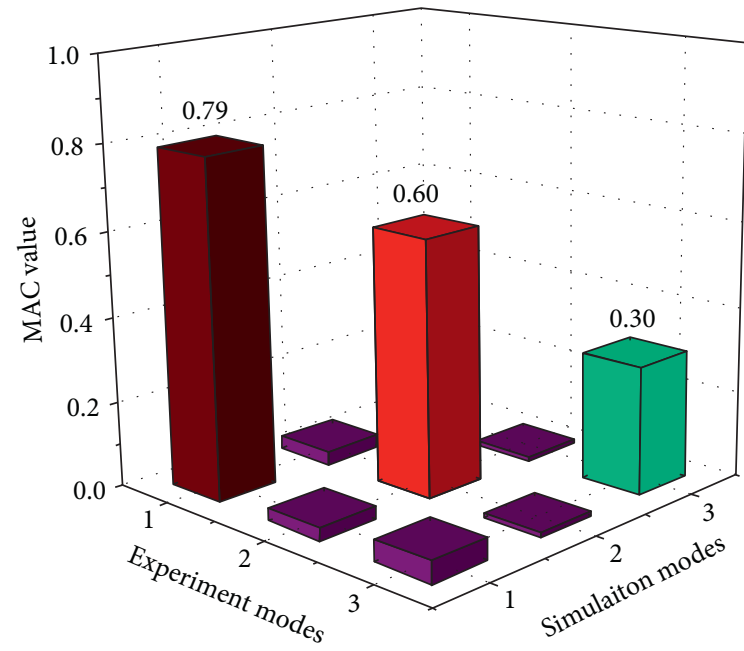

Figure 9: MAC values for the tested and simulated modal shapes.

radiated into vehicle interior. To get the Sound Pressure Level (SPL) near the driver's ear position, PSD of the flow field has been calculated. It was used as an excitation source to the equivalent model, and then the modal superposition FEM was applied for the calculation.

3.1. Calculation of PSD of Flow Field with Corcos Model. The vortices in the turbulent flow near the side glass interact with each other, making the vortices continually generated and shedding. As the vortices shed, large pressure fluctuation can be formed, and the pressure fluctuation in the Turbulent Boundary Layer (TBL) is the main factor that causes the glass vibration and noise radiation. Pressure fluctuation is a stochastic process without deterministic law of change and therefore cannot be described with a definite function. However, it has a certain statistical regularity. Hence, the pressure fluctuation can be characterized with statistical methods with correlation function and PSD. The auto and cross PSD of pressure fluctuation can be calculated with the following equations:

$$
\begin{aligned}
\phi(\omega) & =\int_{-\infty}^{+\infty} E[p(t) p(t+\tau)] e^{-i \omega \tau} d \tau, \\
S\left(\xi_{1}, \xi_{2}, \omega\right) & =\int_{-\infty}^{+\infty} E\left[p_{1}(t) p_{2}(t+\tau)\right] e^{-i \omega \tau} d \tau,
\end{aligned}
$$

where $\omega$ is the angular frequency, $E$ is the desirability function, $p$ is the pressure distribution, $p_{1}$ is the pressure distribution on point $1, p_{2}$ is the pressure distribution on point 2 , and $\xi_{1}, \xi_{2}$ are the distances of two points in $\mathrm{x}$ and $\mathrm{y}$ direction separately.

Surface pressure fluctuation of side glass changes with time and space. Thus, it is difficult to use the above formulas directly. In 1967, Corcos [8] established firstly a classical PSD model of TBL pressure fluctuation. The model is a semiempirical formula, with which the PSD of pressure fluctuation can be estimated with several aerodynamic parameters which can be easily measured. In the Corcos model, the main concerned parameters are frequency and auto and cross PSD. To make the model independent from the dimension, the important parameters in the model are dimensionless treated. The reference frequency and reference PSD can be set, respectively, as follows:

$$
\begin{aligned}
& \omega_{0}=\frac{u_{0}}{\delta^{*}}, \\
& \phi_{0}=\frac{q^{2} \delta^{*}}{u_{0}},
\end{aligned}
$$

where $u_{0}$ is the free stream velocity, $\delta^{*}$ is the boundary layer thickness, and $q=(1 / 2) \rho u_{0}^{2}$ stands for the flow dynamic pressure. The dimensionless frequency and autospectrum density can be described as follows:

$$
\begin{gathered}
\bar{\omega}=\frac{\omega}{\omega_{0}}, \\
\bar{\phi}(\omega)=\frac{\phi(\omega)}{\phi_{0}} .
\end{gathered}
$$

The dimensionless auto PSD can be obtained by fitting the experimental data [9]:

$$
\begin{aligned}
& \bar{\phi}(\bar{\omega})=2.14 \times 10^{-5} \quad \bar{\omega} \leq 0.25 \\
& \bar{\phi}(\bar{\omega})=7.56 \times 10^{-6} \bar{\omega}^{-0.75} \quad 0.25<\bar{\omega} \leq 3.5, \\
& \bar{\phi}(\bar{\omega})=1.27 \times 10^{-4} \bar{\omega}^{-3} \quad 3.5<\bar{\omega} .
\end{aligned}
$$

With the relationship between dimensionless auto PSD and power, the real auto PSD can be estimated. Therefore, a semiempirical formula was proposed for calculating the cross PSD [9]: 


$$
S\left(\xi_{1}, \xi_{2}, \omega\right)=\phi(\omega) e^{-\left|\xi_{1}\right| / u_{\mathrm{c}} / \alpha \omega} e^{-\left|\xi_{2}\right| / u_{\mathrm{c}} / \beta \omega} e^{-i \omega \xi_{1} / u_{\mathrm{c}}},
$$

where $\alpha, \beta$ are exponential coefficients, they can be obtained through experimental fitness. Desirable value for them can be 0.1 and 0.77 , respectively [10]. $u_{c}$ represents the local convective velocity.

In conclusion, only free stream velocity $u_{0}$, boundary layer thickness $\delta^{*}$, and local convective velocity $u_{c}$ are needed for Corcos model to approximately calculate the auto and cross PSD.

\subsection{Solution of Vibroacoustic Coupling with Modal Super-} position Method. To calculate the radiated noise caused by the vibrating side glass to the acoustic cabin, the following assumptions are taken based on the actual situation: the glass is a kind of viscoelastic material, and under wind excitation, only small deformation occurs. The vibrating glass has definite coupling effect with the acoustic cavity of the driver's ear space. Based on the above assumptions, this problem can be regarded as vibroacoustic coupling, so the entire coupling system satisfies the following coupling equation:

$$
\begin{aligned}
& {\left[\begin{array}{cc}
K_{\mathrm{s}}+i \omega D_{\mathrm{s}}-\omega^{2} M_{\mathrm{s}} & C \\
\omega^{2} C^{T} & K_{\mathrm{a}}+i \omega D_{\mathrm{a}}-\omega^{2} M_{\mathrm{a}}
\end{array}\right]\left[\begin{array}{l}
u(\omega) \\
p(\omega)
\end{array}\right]} \\
& \quad=\left[\begin{array}{c}
f_{\mathrm{s}}(\omega) \\
f_{\mathrm{a}}(\omega)
\end{array}\right],
\end{aligned}
$$

where $K_{s}, D_{s}$, and $M_{s}$ are the stiffness, damping, and mass matrix of the structure respectively; $K_{\mathrm{a}}, D_{\mathrm{a}}$, and $M_{\mathrm{a}}$ are the stiffness, damping, and mass matrix of the cavity respectively; $C$ is the coupling matrix; $f_{\mathrm{s}}$ is the structure load vector; $f_{\mathrm{a}}$ is the cavity load vector; $u$ is the structure displacement vector; and $p$ is the cavity pressure vector.

There are two basic FEMs to solve the vibroacoustic coupling problem: modal superposition method and step-bystep integration method [11]. For linear problems, modal superposition is a method with high accuracy and efficiency. The basic idea of modal superposition method is to solve the coupled equations in modal space. For a system in modal space with $\mathrm{N}$ DOFs, the equations can be decoupled, and the response in modal coordinate can be obtained. Then, through linear inverse transformation, the response in physical coordinate can be obtained further. In practice, only a few of the modes in the system are dominant, so only the effects of these main modes are considered. It is not necessary to solve all the equations. By transforming the physical equations into modal space, the number of equations can be reduced, and hence the complexity of the response problem can decrease greatly under random excitation.

In the case without damping, the structure and acoustic cavity satisfy the following formulas:

$$
\begin{aligned}
& \left(K_{\mathrm{s}}-\lambda^{\mathrm{s}} M_{\mathrm{s}}\right) \Psi^{\mathrm{s}}=0, \\
& \left(K_{\mathrm{a}}-\lambda^{\mathrm{a}} M_{\mathrm{a}}\right) \Psi^{\mathrm{a}}=0,
\end{aligned}
$$

where $\lambda^{s}, \Psi^{s}$ are the eigenvalue and eigenvector of structure, respectively, and $\lambda^{\mathrm{a}}, \Psi^{\mathrm{a}}$ are the eigenvalue and eigenvector of acoustic cavity, respectively.

$$
\begin{aligned}
& \Psi^{\mathrm{s}}=\left[\Psi_{1}^{\mathrm{s}}, \Psi_{2}^{\mathrm{s}}, \cdots, \Psi_{m_{s}}^{\mathrm{s}}\right], \\
& \Psi^{\mathrm{a}}=\left[\Psi_{1}^{\mathrm{a}}, \Psi_{2}^{\mathrm{a}}, \cdots, \Psi_{m_{s}}^{\mathrm{a}}\right] .
\end{aligned}
$$

Then,

$$
\begin{aligned}
& u(\omega)=\Psi^{\mathrm{s}} \underline{q}^{\mathrm{s}}(\omega), \\
& p(\omega)=\Psi^{\mathrm{a}} \underline{q}^{\mathrm{a}}(\omega),
\end{aligned}
$$

where $q^{\mathrm{s}}, q^{\mathrm{a}}$ are the displacement and pressure in generalized modal coordinate respectively.

Therefore, in modal space, the coupling equation is transformed into the following form:

$$
\begin{gathered}
{\left[\begin{array}{cc}
\Psi^{\mathrm{sT}}\left(K_{\mathrm{s}}+i \omega D_{\mathrm{s}}-\omega^{2} M_{\mathrm{s}}\right) \Psi^{\mathrm{s}} & \Psi^{\mathrm{sT}} C \Psi^{\mathrm{a}} \\
\Psi^{\mathrm{aT}} \omega^{2} C^{\mathrm{T}} \Psi^{\mathrm{s}} & \Psi^{\mathrm{aT}}\left(K_{\mathrm{a}}+i \omega D_{\mathrm{a}}-\omega^{2} M_{\mathrm{a}}\right) \Psi^{\mathrm{a}}
\end{array}\right]} \\
\cdot\left[\begin{array}{l}
\underline{q}^{\mathrm{s}}(\omega) \\
\underline{q}^{\mathrm{a}}(\omega)
\end{array}\right]=\left[\begin{array}{c}
\Psi^{\mathrm{sT}} \underline{f}_{s}(\omega) \\
\Psi^{\mathrm{aT}} \underline{f}_{\mathrm{a}}(\omega)
\end{array}\right],
\end{gathered}
$$

which is,

$$
\underline{A}(\omega) \underline{q}(\omega)=\underline{f}(\omega) .
$$

The underlined quantities represent that they are in modal space, where,

$$
\begin{aligned}
& \underline{A}(\omega)=\left[\begin{array}{cc}
\Psi^{\mathrm{sT}}\left(K_{\mathrm{s}}+i \omega D_{\mathrm{s}}-\omega^{2} M_{\mathrm{s}}\right) \Psi^{\mathrm{s}} & \Psi^{\mathrm{sT}} C \Psi^{\mathrm{a}} \\
\Psi^{\mathrm{aT}} \omega^{2} C^{\mathrm{T}} \Psi^{\mathrm{s}} & \Psi^{\mathrm{aT}}\left(K_{\mathrm{a}}+i \omega D_{\mathrm{a}}-\omega^{2} M_{\mathrm{a}}\right) \Psi^{\mathrm{a}}
\end{array}\right], \\
& \underline{q}(\omega)=\left[\begin{array}{c}
\underline{q}^{\mathrm{s}}(\omega) \\
\underline{q}^{\mathrm{a}}(\omega)
\end{array}\right], \\
& \underline{f}(\omega)=\left[\begin{array}{c}
\Psi^{\mathrm{sT}} \underline{f}_{\mathrm{s}}(\omega) \\
\Psi^{\mathrm{aT}} \underline{f}_{\mathrm{a}}(\omega)
\end{array}\right] .
\end{aligned}
$$

Let $\underline{H}(\omega)=\underline{A}^{-1}(\omega)$.

Then,

$$
\underline{q}(\omega)=\underline{H}(\omega) \underline{f}(\omega),
$$

where $\underline{H}(\omega)$ is the transfer function in modal space, $f$ is the input, and $\underline{q}$ is the output.

Therefore, the following formula can be obtained in modal space:

$$
S_{\underline{q}}(\omega)=\underline{H}^{*}(\omega) S_{\underline{f}}(\omega) \underline{H}^{\mathrm{T}}(\omega)
$$

where $S_{\mathrm{f}}, S_{\mathrm{q}}$ are the input and output cross PSD in modal space, respectively, and $\underline{H}^{*}(\omega)$ is the conjugate matrix of $\underline{H}(\omega)$.

The input cross PSD can be calculated by the empirical formula of Corcos model, so the output cross PSD can be calculated through Equation (16), and then the radiated SPL can be calculated.

To calculate the SPL near the driver's ear position with FEM, the walls of the acoustic cavity behind the side glass are considered as rigid without vibration. Figure 10 shows the calculated result. 
It can be seen from Figure 10 that there are definite resonance peaks in the position corresponding to the natural frequencies of the structure, especially at the low frequency range. The corresponding frequencies at other peaks are close to the natural frequencies of the acoustic cavity.

\section{Verification of Radiated SPL with Experiment}

To verify the correctness of the above-mentioned method, the simulated results need to be verified by experiment. The usual approach to collect the data of SPL is to do the test with microphone positioned in the vehicle interior at a full-scale aeroacoustic wind tunnel. However, during the experiment, the exterior convective and acoustic pressure fluctuations are mixed together. Hence, the noise measured with microphone interior was the total noise level contributed from these two parts and could not be used for comparative analysis. Therefore, the convective pressure fluctuationinduced vibration test of the side glass was performed. With Laser Vibrometer, the surface vibration velocity distribution of the side glass can be obtained directly. Based on the test data, the radiated sound near the driver's ear position could be calculated with BEM.

\subsection{Vibration Test of the Side Glass with Laser Vibrometer.} Based on the Doppler effect, Laser Vibrometer was applied in the wind tunnel to collect the data of the vibration velocity distribution on the side glass surface at the wind speed $120 \mathrm{~km} / \mathrm{h}$.

Experiment was conducted at the full-scale aeroacoustic wind tunnel of Shanghai Automotive Wind Tunnel Center (SAWTC) with testing equipment produced by Polytec $\mathrm{GmbH}$. Testing equipment (Figure 11) included a laser scanning head model PSV-I-500, data sampling system PSVF-500, and data analysis software PSV. Close to the laser scanning head model PSV-I-500, there stood another reference laser unit to record the reference phase information, which is necessary for the further calculation of sound radiation with BEM. The testing equipment was set out-offlow of the full-scale aeroacoustic wind tunnel, with about 4meter distance perpendicular to the tested side window glass. Data sampling frequency was set to $7.8125 \mathrm{kHz}$. During this test, for every test point (Figure 12), data sampling time was $1.28 \mathrm{~s}$ with 20 times averaging to get a smooth frequency spectrum. After one test point was finished, the laser scanning head could move to the next point automatically. However, velocity distribution was scanned only in the Z-direction which is almost identical with window's normal vector.

4.2. Calculation of the Radiated Noise. The vibration velocity distribution of the side glass was applied as an input condition to calculate the sound radiation near the driver's ear position. Firstly, the acoustic boundary element grid was established according to the spatial distribution of the test points. Then, the vibration velocity spectra of the measured points were imported. Finally, the SPL radiated to the

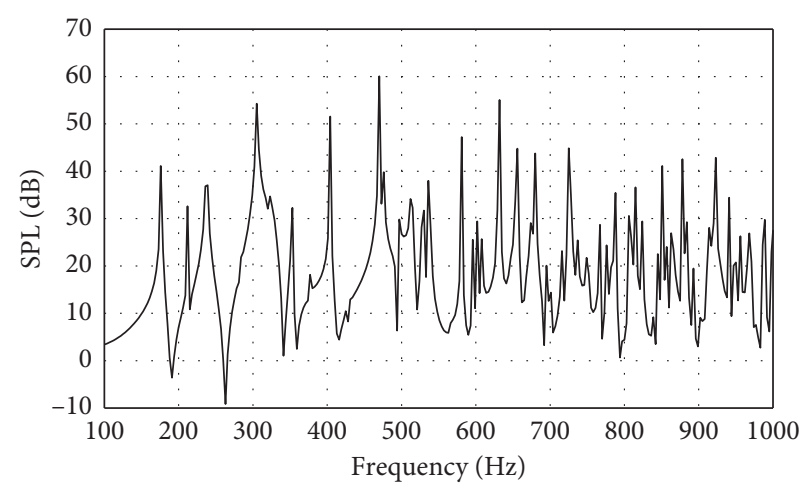

FIGURE 10: Simulation result of radiated SPL near the driver's ear position.

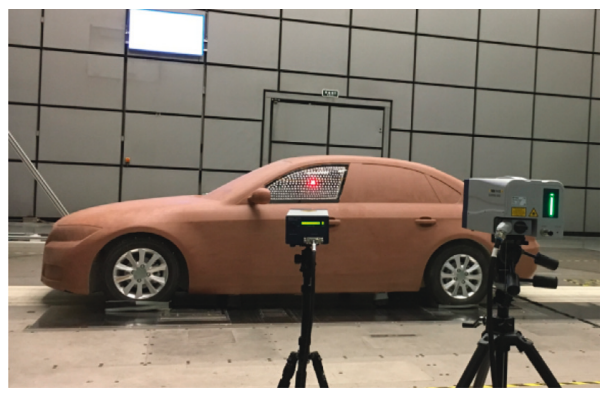

FIGURE 11: Vibration velocity distribution test with Laser Vibrometer in the wind tunnel.

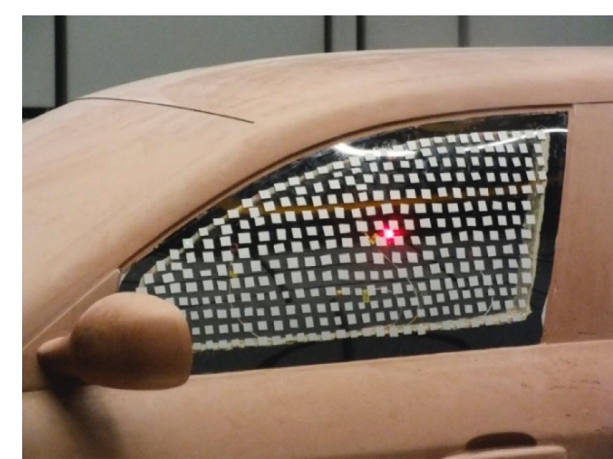

FIGURE 12: Measurement points distribution on the side glass for Laser Vibrometer test.

driver's ear position was calculated with BEM. According to the grid size of the boundary element, the analyzing cut-off frequency was about $1000 \mathrm{~Hz}$. Figure 13 shows the semisimulation (based on the experimental data) result of the radiated noise.

4.3. Comparison of the Radiated SPL. As shown in Figure 14, the simulation result with Corcos model is compared with the semisimulation result. It can be found that these two results are in good agreement. At the low frequency range below $300 \mathrm{~Hz}$, the whole body has much vibration energy during wind tunnel test, which can cause the side glass to 


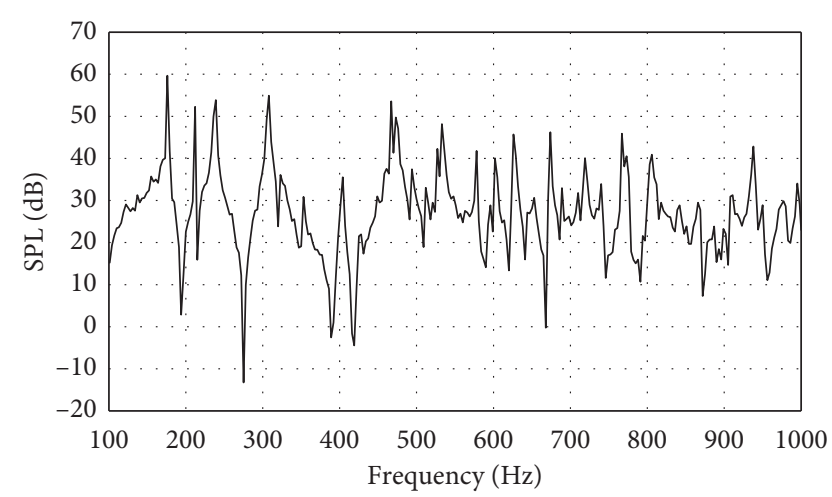

Figure 13: Semisimulation result of the radiated SPL near the driver's ear position.

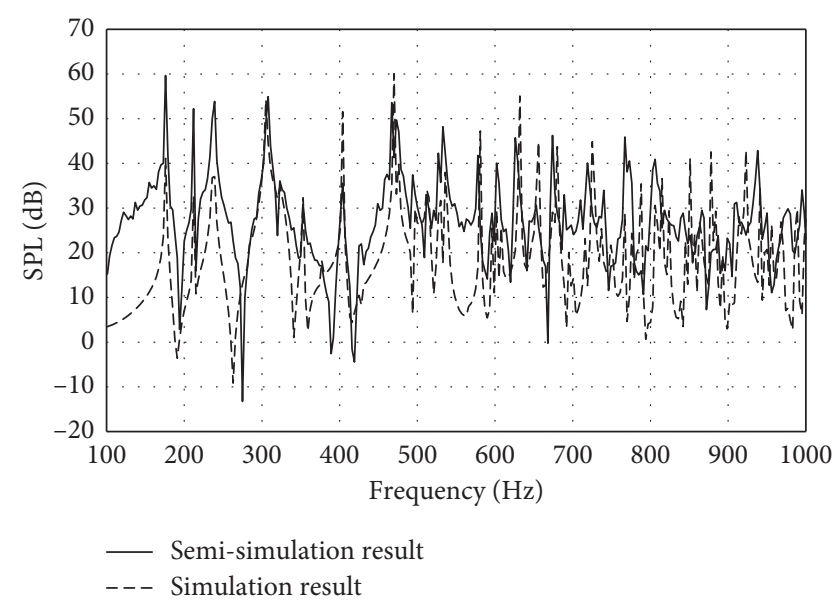

FIGURE 14: Comparison of the radiated noise from semisimulation and simulation.

vibrate. However, the simulation with Corcos model does not include this part of vibration, so that the resonance peaks below $300 \mathrm{~Hz}$ are some lower in comparison with the semisimulation result. At the high frequency range, as the mode density becomes higher, the corresponding frequency of the resonance peaks based on modal superposition has some slight deviation from the result of semisimulation. However, the overall SPL from $500 \mathrm{~Hz}$ to $1000 \mathrm{~Hz}$ of simulation result is $57.5 \mathrm{~dB}$ and for semisimulation is $56.3 \mathrm{~dB}$. Hence, they are in good agreement.

From above comparative analysis, it is proved that it is applicable to calculate flow induced glass vibration and radiated noise of the DrivAer model with Corcos model and modal superposition method.

\section{Conclusions}

(1) The fixed constraint boundary condition of the side glass glued on the DrivAer clay model with some elastic DOFs was set equivalent to a series of springs for the modelling with FEM. To find out the optimal equivalent solution, an optimization platform based on Matlab-Abaqus cosimulation was developed originally and the genetic algorithm was applied to find out the best-fitted spring stiffness with natural frequencies as the optimization target. Through comparison of the simulated and tested modal results, it is demonstrated that natural frequencies and mode shapes are in good agreement. Hence, this equivalent method was validated.

(2) Corcos model and finite element modal superposition method were used to calculate flow-induced side glass vibration and radiated interior noise of the DrivAer model. Through comparison of simulation and semisimulation results, it shows good agreement up to $1000 \mathrm{~Hz}$. It demonstrates that the above method is applicable to calculate the sound radiation caused by the side glass's vibration at the low and middle frequency range. Therefore, an approach of calculating sound radiation of a vibrating glass caused by the air convective pressure fluctuation was explored.

\section{Conflicts of Interest}

The authors declare that there are no conflicts of interest regarding the publication of this paper.

\section{Acknowledgments}

The authors are grateful for the grants from National Natural Science Foundation of China (51575394).

\section{References}

[1] S. Vergne, F. V. Herpe, and J. Viot, "Spatio-temporal analysis of wall pressure fluctuations on several automotive sideglasses," International Journal of Aerodynamics, vol. 1, no. 3-4, pp. 354-372, 2011.

[2] M. Hartmann, J. Ocker, T. Lemke et al., "Wind noise caused by the side-mirror and a-pillar of a generic vehicle model," in Proceedings of AIAA/CEAS Aeroacoustics Conference, Dallas, TX, USA, June 2015.

[3] A. Schell and V. Cotoni, "Prediction of interior noise in a sedan due to exterior flow," SAE International Journal of Passenger Cars-Mechanical Systems, vol. 8, no. 3, pp. 10901096, 2015.

[4] D. Blanchet, A. Golota, N. Zerbib et al., "Wind noise source characterization and how it can be used to predict vehicle interior noise," in Proceedings of 8th International Styrian Noise, Vibration \& Harshness Congress: The European Automotive Noise Conference, Graz, Austria, July 2014.

[5] D. Blanchet, L. Alimonti, A. Golota et al., "Applying complex turbulent cross-correlation function to an sea side glass to predict interior wind noise," in Proceedings of 9th International Styrian Noise, Vibration \& Harshness Congress: The European Automotive Noise Conference, Graz, Austria, June 2016.

[6] A. I. Heft, T. Indinger, and N. A. Adams, "Experimental and numerical investigation of the DrivAer model," in Proceedings of ASME 2012 Fluids Engineering Division Summer Meeting Collocated with the ASME 2012 Heat Transfer Summer Conference and the ASME 2012, International Conference on Nanochannels, Microchannels, and Minichannels, pp. 35493562, Stone Mountain, GA, USA, September 2012. 
[7] R. F. Soares, K. P. Garry, and J. Holt, "Comparison of the farfield aerodynamic wake development for three drivaer model configurations using a cost-effective RANS simulation," in Proceedings of $W_{C X^{\mathrm{TM}}}$ 17: SAE World Congress Experience, Detroit, MI, USA, April 2017.

[8] G. M. Corcos, "The resolution of turbulent pressure at the wall of a boundary layer," Journal of Sound and Vibration, vol. 6, no. 1, pp. 59-70, 1967.

[9] G. M. Corcos, "Resolution of pressure in turbulence," Journal of the Acoustical Society of America, vol. 35, no. 2, pp. 192-199, 1963.

[10] T. S. Miller, J. M. Gallman, and M. J. Moeller, "Turbulent boundary layer models for acoustic analysis," Journal of Aircraft, vol. 49, no. 6, pp. 1739-1754, 2011.

[11] F. Lu, M. Wang, Q. Qu et al., "Analysis of dynamic response of vehicle structures by using the method of mode superposition," Journal of Zibo University: Nature Science and Engineer Edition, vol. 4, no. 4, pp. 70-74, 2002. 


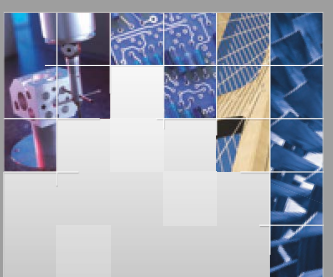

\section{Enfincering}
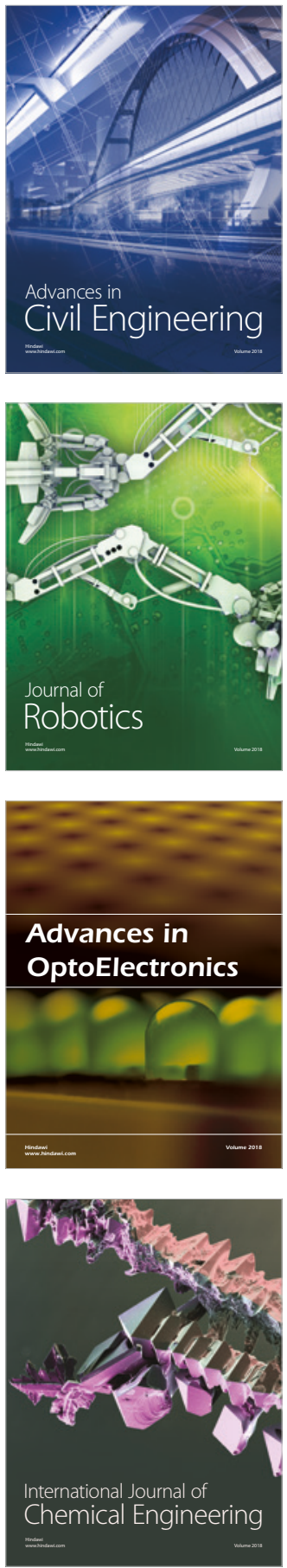

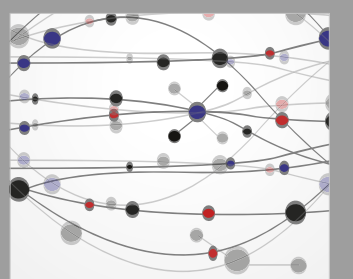

\section{Rotating \\ Machinery}

The Scientific World Journal

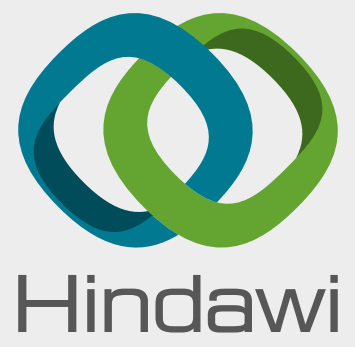

Submit your manuscripts at

www.hindawi.com
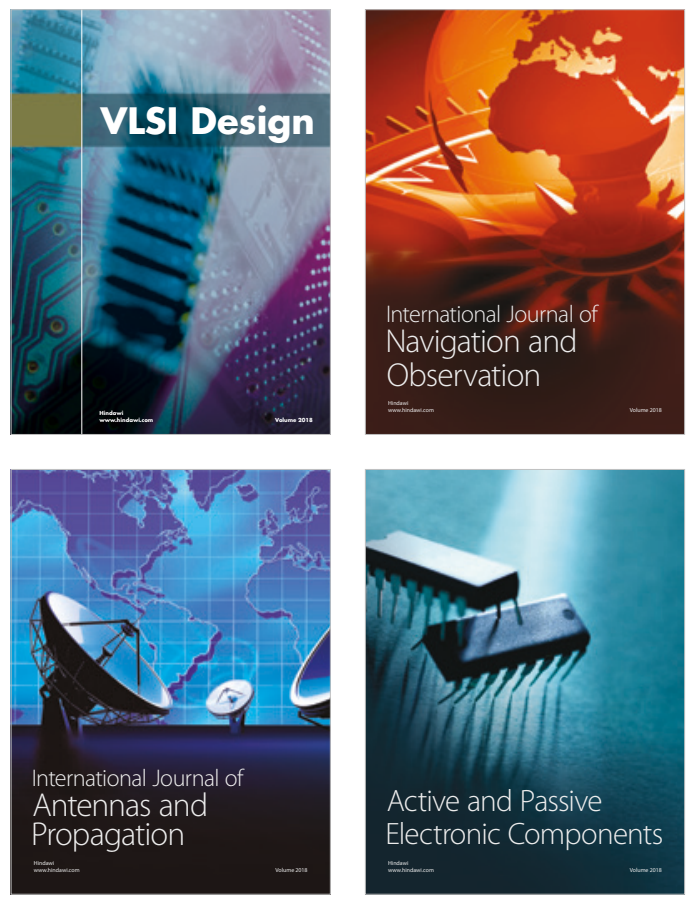
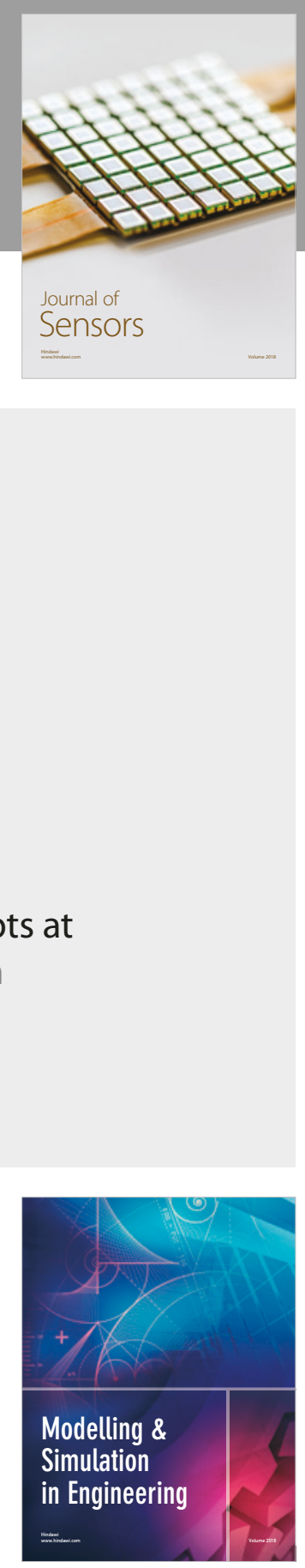

\section{Advances \\ Multimedia}
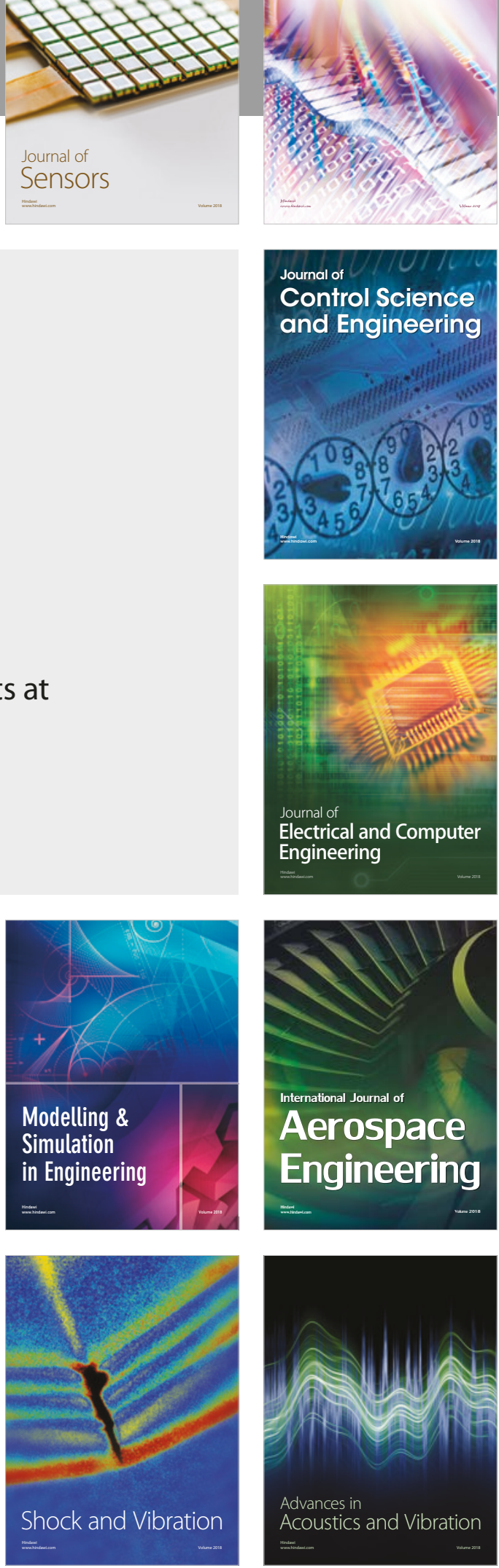